\title{
DETERMINATION OF RADIOLOGICAL PARAMETERS OF SOME ACTIVE PHARMACEUTICAL INGREDIENTS USING WINXCOM SOFTWARE IN THE ENERGY RANGE $1 \mathrm{KeV}$ TO 100GeV
}

\author{
Arome Aruwa ${ }^{1} 凹$, Philibus Musa Gyuk ${ }^{2}$, Columbus Tobechukwu Eze ${ }^{1}$, Suleiman Isah ${ }^{1}$, Achor \\ Mathias Ogwo ${ }^{1}$, Ashibi Martha Ali Adejo ${ }^{1}$ (D) \\ ${ }^{1}$ Department of Science Laboratory Technology, Federal Polytechnic, Idah, Nigeria \\ ${ }^{2}$ Department of Physics, Kaduna State University, Kaduna, Nigeria
}

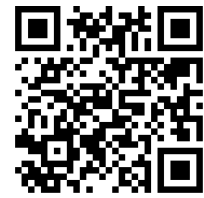

\section{ABSTRACT}

The effective atomic number, electron density and mass attenuation coefficient of some selected active pharmaceutical ingredients such as Diclofenac Sodium, Femotidine, Alprazolam, Amiodar, Amiodarone, Ciprofloxacin, and Nimesulide have been calculated over the energy range from $1 \mathrm{keV}$ to $100 \mathrm{GeV}$ for total and partial photon interactions by using WinXCom. The obtained data shows that the change in mass attenuation coefficient and electron density varies with energy and chemical composition of the active pharmaceutical ingredients (API's) in drugs. The results in the variation of photon interaction with energy and effective atomic number of the API's in drug are shown in the logarithmic graphs.

Received 21 August 2021

Accepted 08 September 2021

Published 31 October 2021

\section{CorrespondingAuthor}

Arome Aruwa, aroaruwa@gmail.com DOI 10.29121/IJOEST.v5.i5.2021.208

Funding: This research received no specific grant from any funding agency in the public, commercial, or not-for-profit sectors.

Copyright: (C) 2021 The Author(s). This is an open access article distributed under the terms of the Creative Commons Attribution License, which permits unrestricted use, distribution, and reproduction in any medium, provided the original author and source are credited.

Keywords: Effective Atomic Number, Electron Density, Active Pharmaceutical Ingredient's, Winxcom, Pharmaceutical, Mass Attenuation Coefficient

\section{INTRODUTION}

Mass Attenuation Coefficients $(\mu / \rho)$, Effective atomic number (Zeff) and Electron Density (Nel) are important parameters in determining the interactions of X-rays and gamma photons in matter (Manohara and Hanagodimath (2007). By the large applications and extensive use of gammaactive isotopes in medicine, industry and agriculture, the study of absorption of gamma rays with biological materials is essential in radiation medicine and in the medical field. Mass attenuation coefficient is a measure of how strongly a substance absorbs or scatters radiation at a given wavelength per unit mass per unit area. The knowledge of mass attenuation coefficients of X-rays and gamma photons in biological and other important materials is of significant interest for industrial, biological, agricultural and medical applications (Jackson and Hawkes (1981). In composite materials, the energy delivered through the photon interactions, a single number cannot represent the atomic number uniquely across the entire energy range, as in case of pure elements. This number for composite materials is known as "effective atomic number" (Zeff) and it varies with energy as pointed out by G. J. Hyne (Hine (1952). 
On the other hand, the concept of z-dependence of photon attenuation coefficient has been utilized in many applications of radiation studies. And it is very important to evaluate the amount of radiation especially in medical Physics (Shivalinge et al. (2004), Ingalagondi et al. (2017). Additionally, the mass attenuation coefficient provides a wide variety of information about fundamental properties of the matter in the atomic and molecular level. Accurate values of the photon mass attenuation coefficients are required to provide essential data in diverse fields such as nuclear diagnostics (computerized tomography), radiation protection, nuclear medicine, radiation dosimetry, gamma ray fluorescence studies, and radiation biophysics. The mass attenuation coefficients are also widely used in the calculation of the photon penetration and the energy deposition in biological shielding and other dosimetric materials.

The application of such effective atomic number can be described in two ways (i) the effective atomic number can be put into formulae and in this way a compound can be reduced to an ordinary element, if calculation of Z-dependent effects are to be carried out and (ii) the effective atomic number can be used to find compound with atomic composition like air or water, etc. Hence, a precise knowledge of effective atomic number plays a very important role in medical radiation dosimetry, radiation therapy etc. There are many researchers who performed research in determination of effective atomic numbers of composite materials ( Shivaramu et al. (2001), Kiran and Venkata (1997), energies close to absorption edges of the elements (Mayneord (1937), ( Shivaramu et al. (2001), Kuran and Venkata (1997), Teli et al. (1996). In the recent years, several theoretical experimental and investigations have been carried out to understand the nature of interaction of different biological molecules such as, amino acids, fatty acids, proteins, carbohydrates etc., but there are few reports in literature survey on the pharmaceutical active ingredients. Active Pharmaceutical Ingredient (API) is a substance used in a finished pharmaceutical product (FPP), intended to furnish pharmacological activity or to otherwise have direct effect in the diagnosis, cure, mitigation, treatment or prevention of disease, or to have direct effect in restoring, correcting or modifying physiological functions in human beings, the basic functioning product in the drug (Manjunath and Kerur (2015). But the drug which is available in the market is composition of active and inactive pharmaceutical gradients. The aim of this work is to calculate the total attenuation cross sections as well as the composition dependent quantities such as effective atomic number ( $\left.\mathrm{Z}_{\mathrm{eff}}\right)$ and effective electron densities $\left(\mathrm{N}_{\mathrm{e}}\right)$ of active pharmaceutical ingredients and to have a full understanding of the nature of interaction of active pharmaceutical ingredients (API) over some energy range.

\section{METHODOLOGY}

A narrow beam of mono-energetic photons in the X-ray or gamma ray region is attenuated to an intensity $I$ from an incident intensity $I_{0}$ in passing through a material thickness with mass per unit area $\mathrm{x}$, according to the well established BeerLambert's exponential law

$I / I_{O}=\exp \left(-\frac{\mu}{\rho}\right) x$

Equation 1 


$$
\left(\frac{\mu}{\rho}\right)=x^{-1} \ln \left(I / I_{O}\right)
$$

Equation 2

in which $\mu / \rho$ is the mass attenuation coefficient and can be obtained from the measured $\mathrm{I}_{\mathrm{I}} \mathrm{I}_{0}$ and $\mathrm{x}$ data. The photon mass attenuation coefficient for any chemical compounds or a mixture can be written as

$$
\mu / \rho=\sum_{i} w_{i}(\mu / \rho)_{i}
$$

Equation 3

Equation 2 is closely related to the total cross section per atom $\sigma_{\text {tot }}$ according to the relation

$$
\mu / \rho=\sigma_{\text {tot }}\left(N_{A} / M\right)
$$

Equation 4

in which $\mathrm{N}_{\mathrm{A}}$ is Avogadro's number and $\mathrm{M}$ is the atomic weight. The total cross section $\sigma_{\text {tot }}$ in turn, can be written as the sum over contribution from the principal of interactions.

$$
\sigma_{\text {tot }}=\sigma_{\text {tot }}+\sigma_{\text {incoh }}+\pi+k+\sigma_{\text {ph.n. }}
$$

Equation 5

in which $\sigma_{\text {coh }}$ and $\sigma_{\text {comt. }}$ are the coherent (Rayleigh) and incoherent (Compton) scattering cross section, respectively, $\tau$ is the atomic photoelectric cross section, $\mathrm{k}$ is the positron electron pair-production (including triplet) cross section and $\sigma_{\text {ph.n. }}$ is the photonuclear cross section.

The effective (average) atomic cross section $\left(\sigma_{a}\right)$ can be easily determined from the following expression,

$$
\sigma_{a}=\frac{1}{N_{A}} \sum f_{i} A_{i}(\mu / \rho)_{i}
$$

Equation 6

Similarly, effective electronic cross section $\left(\sigma_{\mathrm{e}}\right)$ for the individual element is given by the following relation,

$$
\sigma_{e}=\frac{1}{N_{A}} \sum \frac{f_{i} A_{i}}{Z_{i}}(\mu / \rho)_{i}=\frac{\sigma_{a}}{Z_{e f f}}
$$

Equation 7

Where, $f_{i}$ and $Z_{i}$ are fractional abundance and atomic number respectively of constituent element i. Now the effective atomic number can be written as

$$
Z_{e f f}=\frac{\sigma_{a}}{\sigma_{e}}
$$

Equation 8

The effective electron density $\left(\mathrm{N}_{\mathrm{el}}\right)$ which is the number of electrons per unit mass can be derived by using the Equation 3 and (7), 


$$
N_{e l}=\frac{(\mu / \rho)}{\sigma_{e}}=\frac{N_{A}}{M} Z_{e f f} \sum_{i} n_{i}
$$

Equation 9

The mass attenuation coefficient values can be found in the tabulation by Hubbell and Seltzer (Hubbell and Seltzer (1995). The effective atomic numbers for mixture or composite materials were determined by the formula Jackson and Hawkes (1981). A convenient alternative to manual calculations, using tabulated data, is to generate data as needed, using a computer. For this, Berger and Hubbell (1987) developed a computer program, XCOM, for calculating cross sections and attenuation coefficients for any elements, compounds or mixtures at energies from $1 \mathrm{keV}$ to $100 \mathrm{GeV}$. The program has since undergone a number of updates and now available in window version. Recently, this well-known and much used program has been developed to the Windows platform (Gerward et al. (2001), Gerward et al. (2004), Manjunath and Kerur (2015) and the Windows version is being called WinXCom Manjunath and Kerur (2015), Kadir Günoğlu (2018).

\section{RESULTS AND DISCUSSION}

In this work, the variation of effective atomic number, effective electron density and mass attenuation coefficient with photon energy $1 \mathrm{keV}$ to $100 \mathrm{GeV}$ for seven active pharmaceutical ingredients were studied and the details of the drugs are tabulated in the Table 1 . The results obtained clearly support the remarks made by Hine (1952) that the effective atomic number varies with energy. The chemical compositions of the seven API drugs are organic elements only but the ratio of content is different. Except in Alprazolam which contains Carbon, Hydrogen, Chlorine, and Nitrogen other drugs contain basic organic elements $(\mathrm{C}, \mathrm{H}, \mathrm{O})$, since Alprazolam is grouped in the steroidal class of drug and others are Non steroidal class o drug/Non-Steroidal Anti Inflammatory Class of drug Manjunath and Kerur (2015). The $Z_{\text {eff }}$ values of seven organic materials composed of $\mathrm{H}, \mathrm{C}, \mathrm{N}$, and $\mathrm{O}$ were calculated according to the Equation 1. The obtained results of effective atomic number and total mass attenuation coefficient and are shown in Figure 1, Figure 2, Figure 3.

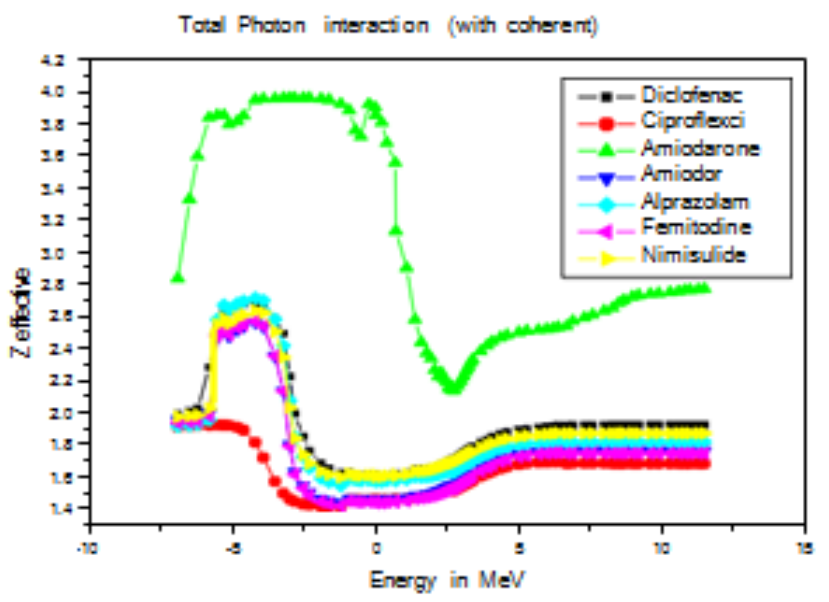

Figure 1 Variation of effective atomic number Zeff of API's in drugs with energies for total photon interaction 
The interpretations of variations are being due to photoelectric effect which varies as $\mathrm{Z}_{4-5}$ and less but significantly due to coherent scattering which varies as $\mathrm{Z}_{2 \text { - }}$ 3. In the intermediate energy region, where incoherent scattering is dominating process, the mass attenuation coefficient is found to be constant and is due to Zdependence of incoherent scattering and significant role played by pair production. Singh (2014) also found the negligible variation between $165 \mathrm{keV}$ and $7 \mathrm{MeV}$ for biological materials. In the high energy region, significant variation in the mass attenuation coefficient is due to the $\mathrm{Z}_{2-3}$ dependence of pair production.

The variation of effective atomic number with photon energy for total photon interactions (Figure 3) which involves a dominating interactions viz., photoelectric, coherent and incoherent processes. The variation of effective atomic number ( $\left.\mathrm{Z}_{\mathrm{eff}}\right)$ with energy is almost similar in case of Amiodar, Femotidine, Nimesulide and Diclofenac Sodium drugs except in the case of Ciprofloxacin and Amiodarone. The discrimination among the effective atomic numbers for the opted API drugs is due to near absorption edges. There were no edge effect observed in Ciprofloxacin drug while two Amiodarone has Iodine $\mathrm{K}, \mathrm{L}_{1}, \mathrm{~L}_{2}, \mathrm{~L}_{3}$ and $\mathrm{M}_{1}$ absorption edges at 34.5, 5.15, $4.76,4.48$ and $1.09 \mathrm{keV}$ respectively. Up to $16-22 \mathrm{keV}$ onwards there is a sharp decrease in effective atomic number and decrease in $\mathrm{Z}_{\text {eff }}$ with energy up to $150 \mathrm{keV}$, showing that contribution of scattering processes increases which decreases $Z_{\text {eff. }}$. From $155 \mathrm{keV}$ to $3.5 \mathrm{MeV}, \mathrm{Z}_{\text {eff }}$ is almost independent of energy. This may be due to the dominant of incoherent scattering in this region. From $3.5 \mathrm{MeV}$ to $410 \mathrm{MeV}$, there is regular increase in $Z_{\text {eff }}$ with photon energy. Hence it is observed that the variation of $Z_{\text {eff }}$ also depends on the relative proportion and the range of atomic numbers of the elements of which API drug is composed (Fig. 1). The Amiodarone has large range of atomic numbers (Z's) from Hydrogen (1) to Iodine (53) than any other API drugs to which the variation in its $Z_{\text {eff }}$ with energy is significant in comparison to any other API's. Variation of Zeff with photon energy for photo electric absorption is shown in the Figure 2 which indicates that composition is also very important as explained above. There is a sudden jump observed in all the cases except in Ciprofloxacin. It has a least range of atomic numbers from $1(\mathrm{H})$ to 9(Fluorine) and hence no absorption edge effect is exist. Diclofenac sodium takes an immediate jump in Zeff at $1.07 \mathrm{keV}$ and $2.82 \mathrm{keV}$, which are the $\mathrm{K}$ absorption edge energies of Sodium ( $\mathrm{Na}$ ) and Chlorine ( $\mathrm{Cl}$ ) respectively. Up to $1 \mathrm{MeV}$ increases sharply and then onwards remains a constant and this is due the fact that photoelectric is the predominant processes in the low energy region $(<1 \mathrm{MeV})$ and is for low $\mathrm{Z}$ materials. The Figure 2 also confirms that the variation of $\mathrm{Z}_{\text {eff }}$ in pharmaceutical drugs probably due to more number of elements in Amiodarone and also the API's having edge effect because Ciprofloxacin has no edges in it. Hence in all other active pharmaceutical ingredients, the variation of $\mathrm{Z}_{\text {eff }}$ is almost independent of energy. This is because of the fact that these API's consist of elements which are same in the number and are of close to the atomic number. 


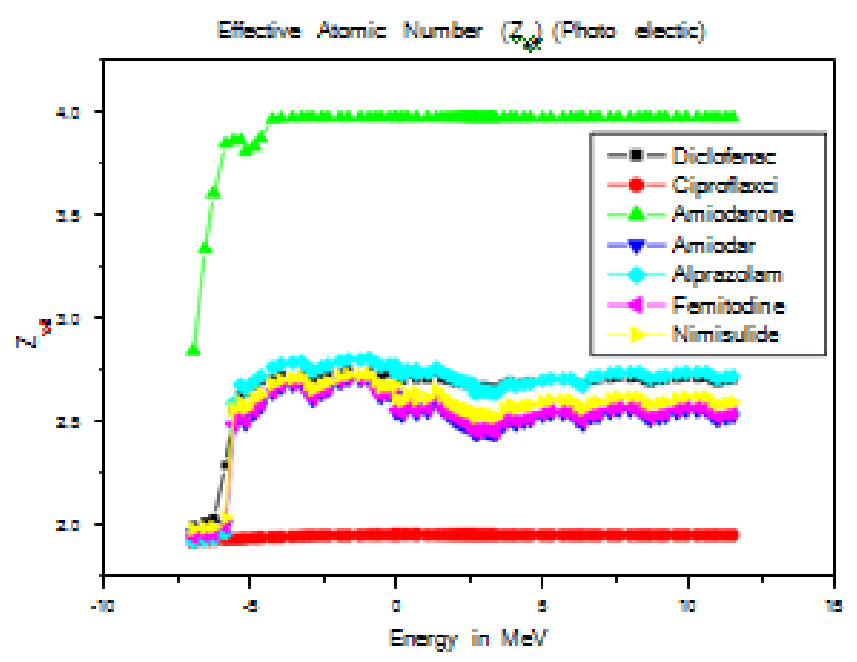

Figure 2 Variation of effective atomic number Zeff of API's in drugs with energies for total photon interaction

The electron density of the selected API's in drug samples are found to be varying from $2.96 \times 10^{23}$ to $10.14 \times 10^{23}$ electrons/g but in the case of Amiodarone it is from $4.85 \times 10^{23}$ to $31.42 \times 10^{23}$ electron/g. Hence electron density is closely related to the effective atomic number and depends on the photon energy and chemical content of the API's in drug samples.

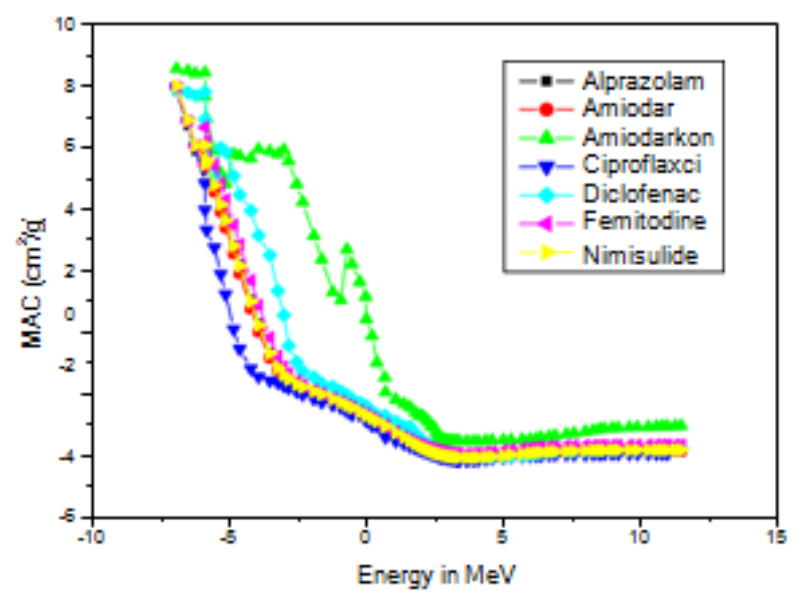

Figure 3 Variation of mass attenuation coefficient with energy for API in drugs

In Figure 3 the results of the total mass attenuation coefficients of active pharmaceutical ingredients against the photon energy is presented. In these cases no absorption edge is found in the ciprofloxacin drug. In the case of Nimesulide, Femotidine, Amiodar, there are two values for mass attenuation coefficients at 2.50 $\mathrm{keV}$ due to K-absorption edge. The value $2.17 \times 10^{2} \mathrm{~cm}^{2} / \mathrm{g}, 2.21 \times 10^{2} \mathrm{~cm}^{2} / \mathrm{g}$ and $2.26 \times 10^{2} \mathrm{~cm}^{2} / \mathrm{g}$ respectively are valid immediately below the absorption edge and $6.57 \times 10^{2} \mathrm{~cm}^{2} / \mathrm{g}, 4.34 \times 10^{2} \mathrm{~cm}^{2} / \mathrm{g}$ and $5.13 \times 10^{2} \mathrm{~cm}^{2} / \mathrm{g}$ respectively are valid immediately above the absorption edge. Alprazolam at $2.89 \mathrm{keV}$ have the values of mass attenuation coefficients $1.43 \times 10^{2} \mathrm{~cm}^{2} / \mathrm{g}$ and $3.12 \times 10^{2} \mathrm{~cm}^{2} / \mathrm{g}$ below and above the chlorine $\mathrm{K}$ absorption edges respectively. Diclofenac sodium has two $\mathrm{K}$ absorption edges at $1.07 \mathrm{keV}$ for Sodium and $2.89 \mathrm{keV}$ for chlorine. The values of 
mass attenuation coefficients varies between $2.10 \times 10^{2} \mathrm{~cm}^{2} / \mathrm{g}$ and $1.65 \times 10^{2} \mathrm{~cm}^{2} / \mathrm{g}$ immediately below the sodium and chlorine $\mathrm{K}$ absorption edge and $2.39 \times 10^{2} \mathrm{~cm}^{2} / \mathrm{g}$ and $3.92 \times 10^{2} \mathrm{~cm}^{2} / \mathrm{g}$ immediately above the Sodium and Chlorine K-absorption edges. The interesting property of API drug in which we opted is the Amiodarone which has $\mathrm{K}, \mathrm{L}_{1}, \mathrm{~L}_{2}, \mathrm{~L}_{3}$ and $\mathrm{M}_{1}$ absorption edges at 34.3, 6.17, 5.85, 4.56 and $1.09 \mathrm{keV}$. The values of mass attenuation coefficient for these edges are $2.83 \times 10^{2} \mathrm{~cm}^{2} / \mathrm{g}$, $3.03 \times 10^{2} \mathrm{~cm}^{2} / \mathrm{g}, 2.74 \times 10^{2} \mathrm{~cm}^{2} / \mathrm{g}, 1.20 \times 10^{2} \mathrm{~cm}^{2} / \mathrm{g}$ and $4.39 \times 10^{3} \mathrm{~cm}^{2} / \mathrm{g}$ respectively below the absorption edges and $17.35 \mathrm{~cm}^{2} / \mathrm{g}, 5.58 \times 10^{+2} \mathrm{~cm} 2 / \mathrm{g}, 7.56 \times 10^{+2} \mathrm{~cm} 2 / \mathrm{g}$, $4.94 \times 10^{+2} \mathrm{~cm} 2 / \mathrm{g}$ and $5.42 \times 10^{+3} \mathrm{~cm} 2 / \mathrm{g}$ above the absorption edges. The above discussion and graph of mass attenuation coefficient vs. energy shows that there are three processes photoelectric absorption, Compton scattering and pair production which are dominant in the interaction with API in the drug materials. Results of (Kaginelli et al. (2009), Manjunath and Kerur (2015) that the theoretical/calculated values have been obtained without considering the edge effects since the effective atomic numbers are under/over estimated when any elements falls below the absorption edge.

\begin{tabular}{ccc}
\hline \multicolumn{2}{|c}{ Table 1 Some of the common active pharmaceutical ingredients used in the drugs } \\
\hline $\mathrm{S} / \mathrm{No}$ & API Name & Chemical Composition \\
\hline 1 & Nimesulide & $\mathrm{C}_{13} \mathrm{H}_{12} \mathrm{~N}_{2} \mathrm{O}_{5} \mathrm{~S}$ \\
\hline 2 & Alprazolam & $\mathrm{C}_{17} \mathrm{H}_{13} \mathrm{ClN}_{4}$ \\
\hline 3 & Amiodar & $\mathrm{C}_{22} \mathrm{H}_{28} \mathrm{FN}_{3} \mathrm{O}_{6} \mathrm{~S}$ \\
\hline 4 & Amiodarone & $\mathrm{C}_{25} \mathrm{H}_{29} \mathrm{I}_{2} \mathrm{NO}_{3}$ \\
\hline 5 & Ciprofloxacin & $\mathrm{C}_{17} \mathrm{H}_{18} \mathrm{FN}_{3} \mathrm{O}_{3}$ \\
\hline 6 & Diclofenac sodium & $\mathrm{C}_{22} \mathrm{H}_{19} \mathrm{Cl}_{2} \mathrm{~N}_{2} \mathrm{NaO}_{4}$ \\
\hline 7 & Femotidine & $\mathrm{C}_{8} \mathrm{H}_{15} \mathrm{~N}_{7} \mathrm{O}_{2} \mathrm{~S}_{3}$ \\
\hline
\end{tabular}

\section{CONCLUSION}

The unique number named in the composite materials as effective number and it may plays an important role in pharmacology or pharmaceutical industry by means of determining the quality and quantity of the drug materials in which one can also identify the active and inactive ingredients added during the manufacturing /formulation processes of different firms.

\section{REFERENCES}

Bethe, A. and Ashkin, J. (1953) Experimental Nuclear Physics, E. Segre, ed. John Wiley \& Sons: New York.

Berger M. and Hubbell J. H. (1987) XCOM: photon cross sections database, Web Version 1.2. Available:http:// physics.nist.gov/xcom National Institute of Standards and Technology, Gaithersburg, MD 20899, USA. (Originally published as NBSIR 87-3597 "XCOM: Photon Cross Sections on a Personal Computer");. Retrieved from https://doi.org/10.2172/6016002

Berger M. and Hubbell J. H. (1999) XCOM: photon cross sections database, Web Version 1.2. Available:http:// physics.nist.gov/xcom National Institute of Standards and Technology, Gaithersburg, MD 20899, USA. (Originally published as NBSIR 87-3597 "XCOM: Photon Cross Sections on a Personal Computer"). 
Burcu, A. and Salih, Z. E. (2014) The Mass Attenuation Coefficients, Electronic Atomic and Molecular Cross Section and Electron Densities for Compounds of Some Biomedically Important Elements at $59.5 \mathrm{KeV}$. Science and Technology of Nuclear Installations, Vol. 1, 1-8S Retrieved from https://doi.org/10.1155/2014/901465

Gerward, L., Guilbert, N., Jensen, K. B. and Levring, H. (2001) X-ray absorption in matter reengineering XCOM. Radiation Physics and Chemistry, 60, 23-24. Retrieved from https://doi.org/10.1016/S0969-806X(00)00324-8

Gerward, L., Guilbert, N., Jensen, K. B. and Levring H. (2004) WinXCom - a program for calculating X-ray attenuation coefficients. Radiation Physics and Chemistry, 71, 653-654. Retrieved from https://doi.org/10.1016/j.radphyschem.2004.04.040

Hine G. J. (1952) The effective atomic numbers of materials for various gamma ray Intercations. Physics Reviews, 85:725.

Hine G. J. (1952): Secondary electron emission and effective atomic numbers.

Nucleonics (1952), 10(1), 9-15. Retrieved from https://doi.org/10.1253/circj1947.15.9-10_Cover1

Hubbell, J. H. (1999) Review of photon interaction cross section data in the medical and biological context. Physics in Medicine and Biology, 44, 1-22. Retrieved from https://doi.org/10.1088/0031-9155/44/1/001

Hubbell J. H. and Seltzer, S. M. (1995) Tables of X-ray mass attenuation coefficients and mass energy absorption coefficients $1 \mathrm{keV}$ to $20 \mathrm{MeV}$ for elements $\mathrm{Z}=1$ 92 and 48 additional substances of dosimetric interest, NISTIR; P 5632. Retrieved from https://doi.org/10.6028/NIST.IR.5632

Ingalagondi. P. K., Omnath P., Mathapati, G. B. and Shivraj G. G. Sankarappa, T. and Hanagodimath, S. M. (2017), Determination of Mass Attenuation Coefficients, Effective atomic number and Electron Density of Lumefantrine in the Energy Range $1 \mathrm{keV}-100 \mathrm{GeV}$. International Journal of Pure and Applied Researches, 4(1), 42-50

Jackson, D. F. and Hawkes, J. D. (1981), X-ray attenuation coefficients of elements and mixtures. Physics Reports, 70, 169-233. Retrieved from https://doi.org/10.1016/0370-1573(81)90014-4

Johns H. E. (1953), the Physics of Radiation Therapy. M. Friedman, ed. Charles C Thomas, Springfield. Pp 374-385.

Kadir Günoğlu (2018), Determination of the mass attenuation coefficients, effective atomic numbers and effective electron numbers of some concrete containing barites for 511, 835 and $1275 \mathrm{keV}$ gamma rays. European Journal of Science and Technology, 14, 185-188 Retrieved from https://doi.org/10.31590/ejosat.453665

Kaginelli, S. B., Rajeshwari, T. Sharanabasappa, K. B. R. and Anilkumar, S. (2009), Effective atomic numbers and electron density of dosimetric materials. Journal of Medical Physics, 34(1), 176-179.Retrieved from https://doi.org/10.4103/0971-6203.54853

Kiran K. T. and Venkata R. K. (1997), Effective atomic numbers for materials of dosimetric interest. Radiation Physics and Chemistry; 50, 545-553. Retrieved from https://doi.org/10.1016/S0969-806X(97)00089-3

Manjunath, A. and Kerur B. R. (2015), Pharmaceutical Active Ingredients in Drugs through Radiological Parameters. Physical Science International Journal, 7(3), 186 - 191 Retrieved from https://doi.org/10.9734/PSIJ/2015/18029 
Manohara, S. R., \& Hanagodimath, S. M., Studies on effective atomic numbers and electron densities of essential amino acids in the energy range $1 \mathrm{keV}-100$ GeV. Nuclear

Instruments and methods in physics research B, 258, 2007, 321-328. Retrieved from https://doi.org/10.1016/j.nimb.2007.02.101

Mayneord W. V. (1937), Acta-Unio Internationalis Contra Cancrum. 2, 271-282.

Roy, B., Chatterjee, B. K., Roy, S. C., Bhattacharya, N. and Choudhury, N. (1997), Photoelectric cross-section derived from measured total attenuation coefficient of photons near absorption edges, of heavier atoms. Applied Radiation Isotopes; 48, 785-78. Retrieved from https://doi.org/10.1016/S0969-8043(96)00321-1

Shivaramu R., Vijayakumar, R., Rajasekaran, L., and Ramamurthy, N. (2001), Effective atmoic number for photon energy absorption of some low-Z substances of dosimetric interest. Radiation Physics and Chemistry, 62, 371377.Retrieved from https://doi.org/10.1016/S0969-806X(01)00221-3

Shivalinge G., Krishnaveni, S., Yashoda, T., Umesh, T.K. \& Ramakrishna G. (2004), Photon mass attenuation coefficients, effective atomic numbers and electron densities of some thermo luminescent dosimetric compounds, PRAMANA, 63, 529-41. Retrieved from https://doi.org/10.1007/BF02704481

Singh, V. P. and Badiger, N. M. (2014), Gamma ray and neutron shielding properties of some alloy materials. Annals of Nuclear Energy, 64, 301-310 Retrieved from https://doi.org/10.1016/j.anucene.2013.10.003

Spiers, W. (1946), Effective Atomic Number and Energy Absorption in Tissues, British Journal of Radiology, 19, 52 - 63. Retrieved from https://doi.org/10.1259/0007-1285-19-218-52

Teli, M. T. and Chaudhari, M. (1996), The attenuation coefficient of ammonium chloride for $662 \mathrm{keV}$ gamma radiations, measured for dilute solution. Radiation Physics and Chemistry, 47, 531-534.Retrieved from https://doi.org/10.1016/0969-806X(95)00162-Q 